\title{
CONVERGENCE OF THE MOTIVIC ADAMS SPECTRAL SEQUENCE
}

\author{
P.HU, I.KRIZ, K.ORMSBY
}

\section{INTRODUCTION}

In [9], we proved convergence of the motivic Adams spectral sequence for spectra $X$ of finite type (for definition, see Section 2 below) in the (bi)stable homotopy category over $\operatorname{Spec}(F)$ where $F$ is an algebraically closed field of characteristic 0 to the homotopy groups of the 2-completion of $X$. Since that time, a number of people asked how far the argument can be generalized using the same method. Notably, the question came up in discussion at the Conference on Motivic Homotopy Theory in Münster, 2009. The purpose of this note is to answer this question. Specifically, recall that $K_{M}(F)$ denotes Milnor $K$-theory of $F$, and $K_{M W}(F)$ is the Milnor-Witt ring [13], which is canonically isomorphic to $\pi_{0+* \alpha}(S)$. This is the "0-slice" of the motivic stable homotopy groups of the sphere; recall that we write $\pi_{k+\ell \alpha}=\pi_{(k+\ell, \ell)}$. Recall also that there is an element $\eta \in K_{M W}^{1}=\pi_{\alpha}(S)$ such that

$$
K_{M W} / \eta \cong K_{M}
$$

Then, in this note, we prove convergence of the motivic Adams spectral sequence for motivic cell spectra $X$ of finite type to the homotopy groups of the completion of $X$ at $p$ and $\eta$ for any field of characteristic 0 . We further prove that completion at $p$ and $\eta$ in the above statement can be replaced by completion at $p$ under suitable conditions.

Throughout this paper (except where specified explicitly), we shall work in the (bi)stable motivic homotopy category (cf. [13]), i.e. $S^{1}=$ $S^{(1,0)}$ and $S^{\alpha}=S^{(1,1)}$ have inverses with respect to the smash product. Denote by $H \mathbb{Z} / p$ the motivic homology spectrum with coefficients in $\mathbb{Z} / p$, and by $X^{A d}$ the realization of the semi-cosimplicial object

$$
\left(X^{A d}\right)_{n}=X \wedge \underbrace{H \mathbb{Z} / p \wedge \ldots \wedge H \mathbb{Z} / p}_{n}
$$

where co-faces are given by the unit $S \rightarrow H \mathbb{Z} / p . X^{A d}$ is what is referred to as the nilpotent completion of $X$ with respect to $H \mathbb{Z} / p$ by Bousfield $[5]$. 
Denote by $X_{p}^{\wedge}$ the Bousfield localization $L_{M \mathbb{Z} / p} X$ [5] of $X$ at the (pushforward of the) Moore spectrum $M \mathbb{Z} / p$. In a very general context, including the present situation, this is equivalent to

$$
\underset{n}{\operatorname{holim}} X / p^{n}
$$

so there is the usual exact sequence

(2) $\quad 0 \rightarrow \operatorname{Ext}^{1}\left(\mathbb{Z} / p^{\infty}, \pi_{*} X\right) \rightarrow \pi_{*}\left(X_{p}^{\wedge}\right) \rightarrow \operatorname{Hom}\left(\mathbb{Z} / p^{\infty}, \pi_{*-1} X\right) \rightarrow 0$.

In the present motivic context, this is treated explicitly in $[15,9]$. Denote similarly

$$
X_{p, \eta}^{\wedge}=\underset{n}{\operatorname{holim}} X /\left(p^{n}, \eta^{n}\right)
$$

There are canonical maps $X \rightarrow X_{p}^{\wedge}, X \rightarrow X_{p, \eta}^{\wedge}$. We will refer to as completion at $p$ resp. completion at $p, \eta$.

The main result we prove here is the following

Theorem 1. Let $F$ be a field of characteristic 0 . Let $X$ be a cell spectrum of finite type over $\operatorname{Spec}(F)$ (see Section 2 below for definition). Then the natural map

$$
X \rightarrow X^{A d}
$$

is a completion at $p, \eta$. When $p>2$ and $c d_{p}(F)<\infty$ and $-1 \in F$ is a sum of squares, or $p=2$ and $c d_{2}(F[i])<\infty$, then (3) is a completion at $p$.

For the definition of $c d_{p}$, see [17]. At $p=2$, one has the following result, which follows in a trivial way from the Gysin sequence in Galois cohomology associated with a quadratic field extension

$$
\ldots H^{n}(F ; \mathbb{Z} / 2) \longrightarrow H^{n}(F[i] ; \mathbb{Z} / 2) \longrightarrow H^{n}(F ; \mathbb{Z} / 2) \stackrel{[-1]}{\longrightarrow} H^{n+1}(F ; \mathbb{Z} / 2) \ldots
$$

(cf. [2] - here [-1] denotes multiplication by $[-1] \in F^{\times} /\left(F^{\times}\right)^{2}=$ $H^{1}(F, \mathbb{Z} / 2)$.).

Proposition 2. For any field $F$ of characteristic $0, c d_{2}(F[i])<\infty$ if and only if

$$
\begin{aligned}
& \text { There exists a constant } r \text { such that }[-1]: \\
& H^{n}(F, \mathbb{Z} / 2) \rightarrow H^{n+1}(F, \mathbb{Z} / 2) \text { is an isomorphism } \\
& \text { for } n \geq r .
\end{aligned}
$$

Obviously, fields of finite transcendence degree, local fields, number fields and $\mathbb{R}$ are covered for $p=2$ by Theorem 1 . These cases of our present convergence result already have been used in the papers $[8,16]$. 
Corollary 3. Under the assumptions of Theorem 1, there exists a convergent spectral sequence ("motivic Adams spectral sequence") with

$$
E_{2}=\operatorname{Cotor}_{\left(H \mathbb{Z} / p_{*}, H \mathbb{Z} / p_{*} H \mathbb{Z} / p\right)}\left(H \mathbb{Z} / p_{*} X, H \mathbb{Z} / p_{*}\right)
$$

convergent strongly to the homotopy groups of the respective completion of $X$.

Proof: The existence and convergence of the Adams spectral sequence is a formal consequence of Theorem 1 by the "Mittag-Leffler convergence Lemma" of Bousfield and Kan (Lemma 5.6, p. 264 of [3]). The point is that the tower involved in the Adams spectral sequence is actually

$$
\left(X^{(n)}\right)
$$

where $X^{(n)}$ is the homotopy fiber of the canonical map from the relevant completion of $X$ as in Theorem 1 to the $n$ 'th cosimplicial co-skeleton of $X^{A d}$. Thus, by Theorem 1, we have

$$
\text { holim } X^{(n)}=*,
$$

which implies that both that

$$
\lim \pi_{*} X^{(n)}=\lim ^{1} \pi_{*} X^{(n)}=0 .
$$

The identification of the $E_{2}$-term goes back to Adams [1].

\section{Comments:}

1. It is well known $([13,14])$ that in dimension $n \alpha, n>0, \pi_{n \alpha}(S)$ is isomorphic to the Witt ring $W$ and that the effect of completion at $2, \eta$ in these dimensions is the completion of $W$ at its augmentation ideal. It is easy to show that for general fields, this does not coincide with completion at 2 (one example mentioned in [10] is the field $\left.F=\mathbb{Q}\left(x_{1}, \ldots, x_{m}, \ldots\right)[i]\right)$. Therefore, the second statement of Theorem 1 would be false if we omit the assumption (4).

For $p>2$, this issue is even sharper. Completion of $\pi_{* \alpha}\left(S^{0}\right)$ at $p$ is isomorphic to

$$
W_{p}^{\wedge} \oplus K_{M}(F)_{p}^{\wedge} .
$$

To see this, recall that by Morel's structure theorem [14, 13],

$$
\begin{aligned}
& \pi_{n \alpha}\left(S^{0}\right)=W \quad \text { for } n>0 \\
& I^{-n} \times_{I^{-n} / I^{-n+1}}\left(K_{M}\right)_{-n}(F) \text { for } n \leq 0 \text {. }
\end{aligned}
$$


where $I$ is the augmentation ideal of $W$. By induction on $n, W / I^{n}$ is 2torsion (since $2=[-1] \in I$ ), so when completing at $p$ odd, (6) becomes (5). The map $\eta$ induces an isomorphism on the first summand and 0 on the second. Therefore, the completion at $p$ is $\eta$-complete if and only if

$$
W_{p}^{\wedge}=0 .
$$

Referring to [11], if the field $F$ has a real ordering (which is equivalent to -1 not being a sum of squares), then there exists an epimorphism

$$
W \rightarrow \mathbb{Z},
$$

which means that (7) cannot hold. Therefore, completion at $\eta$ is necessary for convergence of the Adams spectral sequence at $p>2$ for any such field.

2. In the following sections, by the statement that "the motivic Adams spectral sequence converges for $X$ " we shall simply mean that $X \rightarrow X^{A d}$ is an equivalence. For essentially formal reasons, the Adams spectral sequence always converges to the homotopy groups of $X^{A d}$, [5], Section 6, also [6].

3. We have, by work of Voevodsky [19],

$$
H \mathbb{Z} / 2_{*}=K_{M} / 2[\theta]
$$

where $\theta$ is an element of dimension $1-\alpha$ (the "Tate twist"). For $p>2$, the element $\theta$ exists only when $F$ has $p$ 'th roots of unity, but for any prime $p$, we have by the Bloch-Kato conjecture proved by Voevodsky [18],

$$
\mathbb{H} \mathbb{Z} / p_{k+\ell \alpha}=\begin{array}{ll}
H^{-\ell-k}(F, \mathbb{Z} / p(\ell)) & \text { for } k \geq 0 \\
0 & \text { else. }
\end{array}
$$

The structure of the dual Steenrod algebra is determined by Voevodsky [20], with a gap filled in [21] (see also [9]). One has ([20], Theorem 12.6):

$$
A_{*}=H \mathbb{Z} / 2\left[\tau_{i}, \xi_{i}\right] /\left(\tau_{i}^{2}=\theta \xi_{i+1}+[-1] \tau_{i+1}\right)
$$

for $p=2$ and

$$
A_{*}=H \mathbb{Z} / p\left[\tau_{i}, \xi_{i}\right] /\left(\tau_{i}^{2}=0\right)
$$

for $p>2$, where the elements $\xi_{i}, i>0$ resp. $\tau_{i}, i \geq 0$ have dimensions $\left(p^{i}-1\right)(1+\alpha)$ resp. $\left(p^{i}-1\right)(1+\alpha)+1$. Actually, as shown in [20], $\left(H \mathbb{Z} / 2_{*}, A_{*}\right)$ is not a Hopf algebra but a Hopf algebroid; in (8), $\theta$ is identified with $\eta_{L} \theta$. If one uses $\eta_{R} \theta$ instead, one gets an additional term, which is why the formula in Theorem 12.6 of [20] looks slightly different. This structure theorem will be needed in the remaining sections. 
4. We stress however that the Adams filtration on homotopy groups can be quite bad. Let, for example, $p=2, F=\mathbb{Q}$ and consider the spectrum $Y$ which is the homotopy cofiber of the sequence

$$
S^{-\alpha} \vee S^{[3] \vee 2} \stackrel{\longrightarrow}{\longrightarrow}
$$

Let $X=Y /(4, \eta)$. Then one can see that the kernel of the canonical onto map

$$
\pi_{* \alpha} X \rightarrow H \mathbb{Z} / 2_{* \alpha} X
$$

is not finitely presented as a $K_{M W}(\mathbb{Q})$-module.

Acknowledgement: We would like to thank Mike Hopkins and Fabien Morel for conversations between the years 2008 and 2010, in which they conjectured the convergence of the motivic Adams spectral sequence for cell spectra of finite type to the completion at $(p, \eta)$ for general fields of characteristic 0. Although no method of proof was suggested, there is no doubt that those conversations contributed to the present paper. We are also thankful to Paul Arne Østvær for comments on an earlier (less general) version of this note, and to Alexander Merkurjev for a reference on Proposition 2.

\section{Cell spectra of finite type}

For most of our purposes, it will be convenient to work directly in the motivic (bi)stable homotopy category $\mathcal{S H}_{F}$ over $F$. From this point of view, attaching cells to a spectrum $X$ means simply to form a homotopy cofiber of a map (in $\mathcal{S H}_{F}$ ) of the form

$$
\bigvee_{i} S^{k_{i}+\ell_{i} \alpha} \rightarrow X
$$

The expressions $1+k_{i}+\ell_{i} \alpha$ will be referred to as the dimensions of the cells. Starting with a point and iterating this construction, we obtain the notion of a (motivic) cell spectrum. A priori we could iterate the construction transfinitely, passing to homotopy colimits at limit ordinals, but commutation of homotopy groups with infinite wedges shows that one can construct any cell spectrum by applying at most $\omega$ steps, and taking homotopy direct limit once. It can also be shown (by formal arguments) that a map between motivic cell spectra is an equivalence if and only if it induces isomorphism on all homotopy groups $\pi_{k+\ell \alpha}$ (for general motivic spectra, such map will be called a very weak equivalence in [9]). Also by formal arguments, for any motivic spectrum $X$, there exists a cell spectrum $X^{\prime}$ and a very weak equivalence $X^{\prime} \rightarrow X$ 
(cf. [7]). We will call a cell spectrum $X k$-connective if $\pi_{m+n \alpha} X=0$ for $m<k$. For a map of cell spectra $f: X \rightarrow Y$, we will call $f$ a $k$-equivalence if its homotopy cofiber is $k+1$-connective.

Definition: A cell spectrum of finite (resp. bounded) type is a cell spectrum $X$ where there exists a $k \in \mathbb{Z}$ such that $X$ has no cells in dimension $m+\ell \alpha, m<k$, and at most finitely many cells in dimension $m+\ell \alpha$ for any $m \in \mathbb{Z}$ (resp. no cells of dimension $m+\ell \alpha$ for $\ell<N_{m}$ where $N_{m} \in \mathbb{Z} \cup\{+\infty\}$ depend only on $m$ ). In the bounded case, we will refer to the numbers $N_{m}$ as bounds for $X$; we require that there exists a $k \in \mathbb{Z}$ such that $N_{m}=+\infty$ for $m<k$.

Analogously, we will say that the homotopy groups $\pi_{*} X$ are of bounded type if $X$ is $k$-connective for some $k \in \mathbb{Z}$ and there exist $N_{m} \in \mathbb{Z} \cup\{\infty\}$ for all $m \in \mathbb{Z}$ such that $\pi_{m+\ell \alpha}(X)=0$ for $\ell<N_{m}$.

A priori, in addition to the notion of finite type, we also have another weaker notion of cell spectrum of weakly finite type, which is a homotopy colimit of spectra of the form $X_{m}$ where we have $X_{-1}=*$, and we have cofiber sequences of the form

$$
Y_{m} \rightarrow X_{m} \rightarrow X_{m+1}
$$

where $Y_{m}$ is a wedge summand of a cell spectrum $Z_{m}$, and the wedge $\bigvee Z_{m}$ is a cell spectrum of finite type. Proposition 15 and comments in the Appendix of [9] imply that $H \mathbb{Z} / p$ is a cell spectrum of weakly finite type (while [9] focuses on the case of an algebraically closed field and $p=2$, the same discussion applies in the general case).

Now, however, we have the following

Lemma 4. A wedge summand $X$ of a cell spectrum $X_{0}$ of finite type is equivalent to a cell spectrum of finite type $X^{\prime}$ such that for every cell of $X^{\prime}$ of dimension $k+\ell \alpha$, there exists a $k^{\prime}<k$ and a cell of $X_{0}$ of dimension $k^{\prime}+\ell \alpha$.

Proof: A classic Eilenberg swindle. Let

$$
X_{0} \simeq X \vee Y .
$$

Let $X_{1}$ be the cofiber of the map

$$
X_{0} \rightarrow X_{0}
$$

which is $I d$ on $Y$ and 0 on $X$. Then

$$
X_{1} \simeq X \vee \Sigma X
$$


Let $X_{2}$ be the cofiber of the map

$$
\Sigma X_{0} \rightarrow X_{1}
$$

which is $I d$ on $\Sigma X$ and 0 on $\Sigma Y$. Then

$$
X_{2} \simeq X \vee \Sigma^{2} Y
$$

Iterating this procedure, we obtain a spectrum of finite type equivalent to $X$, satisfying the dimensional condition stated.

Corollary 5. A cell spectrum of weakly finite type is equivalent to a cell spectrum of finite type.

Proof: Use Lemma 4 successively on the spectra $Y_{m}$ in the definition of a cell spectrum of weakly finite type.

Thus, we have

Lemma 6. The spectrum $H \mathbb{Z} / p$ is equivalent to a spectrum of finite type.

Now suppose we have a cell spectrum $X$ and a number $k \in \mathbb{Z}$ such that $X$ is $k$-connective. Then it is easy to see that there exists an equivalent cell spectrum $X^{\prime}$ with no cells of dimension $m+\ell \alpha, m<k$. In fact, $X^{\prime}$ can be obtained in $\omega$ steps where in $i^{\prime}$ th step, $i \in \omega$, we attach cells in dimension $m+i+* \alpha$ - simply attach to $X$ all cells necessary to cancel homotopy in that dimension, and in the end take homotopy fiber of the canonical map from $X$ - here we are using the fact [13] that

$$
\pi_{m+\ell \alpha} S=0 \text { for } m<0 .
$$

We must be substantially more careful to prove an analogous statement for cell spectra of finite type. To this end, we will need a process called "cell cancellation".

Lemma 7. Let $X$ be a $k$-connective motivic cell spectrum of finite (resp. bounded) type. Then there exists an equivalent cell spectrum of finite (resp. bounded) type $X^{\prime}$ such that $X^{\prime}$ has no cells in dimension $m+\ell \alpha, m<k$.

Proof: Let us first consider the finite type case. As remarked above, we may assume $X$ is constructed in $\omega$ steps. Let $i$ be the first step in which one of the cells $e$ attached has dimension $m+\ell \alpha, m<k$ (if no such $i$ occurs, we are done). By (10), the attaching map of $e$ is 
homotopic to 0. Since we already remarked that attaching cells can be interpreted as an operation in the motivic stable homotopy category, we may assume (without changing the dimensions of the cells constituting $X$ ) that the attaching map of $e$ is actually 0 . This gives us a map

$$
S^{m+\ell \alpha} \rightarrow X .
$$

Let $X_{1}$ be the homotopy cofiber of the map (11). Note carefully that $X_{1}$ can be constructed by following the construction of $X$ while "omitting" the cell $e$, i.e. that $X_{1}$ is equivalent to a cell spectrum of finite type with cells of the exact same dimensions as the cells of $X$ with the exception of the cell $e$, which is omitted.

Now note however that by our assumption about $X$, the map (11) must be trivial, i.e. we must have

$$
X_{1} \simeq X \vee S^{m+1+\ell \alpha} .
$$

We see then that by attaching a single cell of dimension

$$
m+2+\ell \alpha
$$

to $X_{1}$ whose attaching map is the injection to the second wedge summand (12), we obtain a cell spectrum equivalent to $X$ with cells in the exact same dimensions except the cell $e$, which is replaced by a cell of dimension greater by 2 .

By iterating this procedure, we clearly eventually eliminate all cells of dimension $m+\ell \alpha, m<k$.

In the bounded type case, the argument is identical. Limit steps, which are required in this case, are filled in by taking direct limits.

Lemma 8. Let $X$ be a cell spectrum such that for every $n$, there exists a cell spectrum $X_{n}$ of finite type and an $n$-equivalence

$$
X_{n} \stackrel{\phi_{n}}{\longrightarrow} X \text {. }
$$

Then $X$ is equivalent to a cell spectrum of finite type.

Proof: By an analogue of Whitehead's theorem (which is true for formal reasons in the present situation, cf. [7]), we get homotopy commutative diagrams of the form

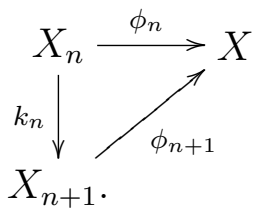


It follows that an induced map

$$
\operatorname{holim} X_{n} \rightarrow X
$$

is an equivalence. Further, the homotopy cofiber of $k_{n}$ must be $n$ connective by the assumption on $\phi_{n}$. By Lemma $7, X_{n+1}$ can be obtained from $X_{n}$ by attaching finitely many cells in each dimension $m+* \alpha, m \geq n$. We deduce that

$$
\text { holim } X_{n}
$$

is a cell spectrum of finite type.

Comment: There is no harm in turning around the arrows in the assumption (13): If we assume instead we have an $n$-equivalence

$$
X \stackrel{f}{\longrightarrow} X_{n},
$$

by the Whitehead theorem, if we denote by $X_{n-1}^{\prime}$ the cell spectrum of finite type obtained from $X_{n}$ by omitting any cells of dimension $(\geq n)+* \alpha$ and by

$$
\iota: X_{n-1}^{\prime} \rightarrow X_{n}
$$

the canonical map, there exists a map (in the homotopy category) $g: X_{n-1}^{\prime} \rightarrow X$ such that

$$
f g \simeq \iota .
$$

Further, $\iota$ is an $n-1$-equivalence, and hence so is $g$.

For completeness, we also note

Lemma 9. A homotopy cofiber of a map of $k$-equivalences is a $k$ equivalence, and a homotopy direct limit of a sequence of $k$-equivalences is a k-equivalence.

Proof: For the first statement, consider a diagram of cofibration sequences

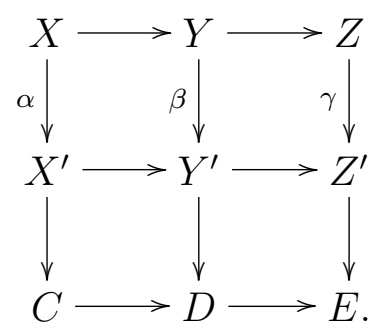


Then $\alpha$ and $\beta$ are $k$-equivalences if and only if $C, D$ are $(k+1)$ connective. But then $E$ is $(k+1)$-connective by the long exact sequence of homotopy groups. For the second statement, simply note that isomorphisms and surjections are preserved by direct limits of sequences.

\section{Convergence}

Lemma 10. 1. The canonical map

$$
S \rightarrow H \mathbb{Z} / p
$$

is a 0-equivalence.

2. For $p=2$, the canonical map

$$
S /(2, \eta) \rightarrow H \mathbb{Z} / 2
$$

is a 1-equivalence. For $p>2$, the canonical map

$$
\iota: S /(p, \eta) \rightarrow H \mathbb{Z} /(p, \eta) \simeq H \mathbb{Z} / p \vee \Sigma H \mathbb{Z} / p
$$

is a 1-equivalence.

Proof: To prove the first statement, the map (15) realizes in homotopy groups the projection

$$
K_{M W}(F) \rightarrow K_{M}(F) / p
$$

$([13,14])$, which is of course onto.

To prove the second statement, for $p=2$, we have

$$
\pi_{* \alpha}(S /(2, \eta))=K_{M W}(F) /(2, \eta)=K_{M}(F) / 2=H \mathbb{Z} / 2_{* \alpha},
$$

and the isomorphism is induced by (16). Using the multiplicative structure of the Milnor-Witt ring, to prove the onto part of the statement, it suffices to prove that the generator

$$
\theta \in \pi_{1-\alpha} H \mathbb{Z} / 2
$$

lifts to

$$
\theta^{\prime} \in \pi_{1-\alpha}(S /(2, \eta)) \cong \mathbb{Z} / 2 .
$$

Now using the pushforward, it suffices to produce $\theta^{\prime}$ for the field $F=\mathbb{Q}$. In that case, we have

$$
2[-1]=0 \in K_{M}(\mathbb{Q})
$$

so

$$
0 \neq[-1] \in \operatorname{Im}\left(\beta: \pi_{1-\alpha} H \mathbb{Z} / 2 \rightarrow \pi_{-\alpha} H \mathbb{Z}\right) .
$$

So we must have

$$
[-1]=\beta \theta
$$


Now note that in $K_{M W}$,

$$
[-1](2+[-1] \eta)=0
$$

SO

$$
2[-1]=0 \in \pi_{-\alpha}(S / \eta) .
$$

Thus, again, $[-1] \in \pi_{-\alpha}(S / \eta)$ satisfies

$$
[-1] \in \operatorname{Im}\left(\beta: \pi_{1-\alpha}\left(S /(2, \eta) \rightarrow \pi_{-\alpha} S / \eta\right),\right.
$$

and we can let $\theta^{\prime}$ be any element of $\pi_{1-\alpha}(S /(2, \eta))$ such that $\beta \theta^{\prime}=[-1]$.

For $p>2$, this method works when $F$ contains $p^{\prime}$ th roots of unity, but otherwise the Tate twist device is not available. However, instead, we may proceed as follows. Consider the diagram

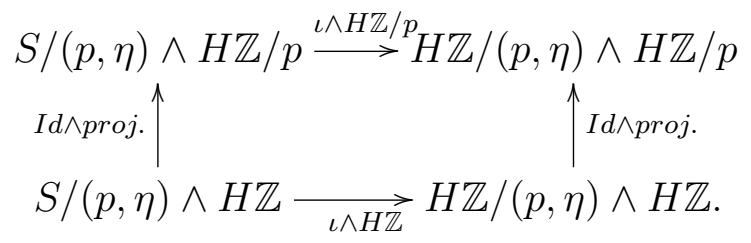

By (9), the top row is a 1-equivalence, but by the fact that $p$ annihilates the bottom row, the verticals are homotopy retracts, so the bottom row is also a 1-equivalence. Now the cofiber $C \iota$ is a cell spectrum of finite type such that

$$
0=\eta: C \iota \rightarrow C \iota .
$$

Suppose $C \iota$ is $k$-connective but not $k+1$-connective for some $k$. We have

$$
\pi_{k+* \alpha} C \iota=\left(\pi_{k+* \alpha} C \iota\right) \otimes_{\mathbb{Z}[\eta]} \mathbb{Z}=\left(\pi_{k+* \alpha} C \iota\right) \otimes_{K_{M W}} K_{M W} / \eta=H \mathbb{Z}_{k+\ell \alpha} C \iota .
$$

Note that this is a "Künneth-like" argument, but we don't have to discuss the Künneth spectral sequence here; the $k+* \alpha$-homotopy groups of a smash product of a $k$-connective spectrum $X$ and a 0 -connective spectrum $Y$ is always to

$$
\pi_{k+* \alpha} X \otimes_{K_{M W}} \pi_{0+* \alpha} Y
$$

by the long exact sequence in homotopy groups.

Thus, by the fact that the bottom row of (20) is a 1-equivalence, we have $k \geq 2$, as claimed.

Lemma 11. For any $k$-connective motivic cell spectrum $X$ (not necessarily of finite type), $X^{A d}$ is $k$-connective. 
Proof: By Lemma 10 1. and Lemma 9,

$$
X \rightarrow X \wedge H \mathbb{Z} / p
$$

is a $k$-equivalence for any $k$-connective cell spectrum $X$, and hence the homotopy fiber $X_{1}$ of $(22)$ is $k$-connective. Iterating this construction by forming a fiber sequence

$$
X_{n+1} \rightarrow X_{n} \rightarrow X_{n} \wedge H \mathbb{Z} / p,
$$

we obtain by induction that $\underset{n}{\operatorname{holim}} X_{n}$ is $k$-connective, and hence $X^{A d}$, which is the homotopy cofiber of the canonical map

$$
\underset{n}{\operatorname{holim}} X_{n} \rightarrow X
$$

is $k$-connective.

The following lemma is not strictly needed in full generality as a part of our proof, but it is nice to note that we can prove it at this point.

Lemma 12. If $X$ is a motivic cell spectrum of finite type, then the motivic Adams spectral sequence converges for $X \wedge H \mathbb{Z} / p$.

Proof: The statement is true for $X=S$ by calculation, so it is true for a cell spectrum with finitely many cells. Now let $X_{(n)}$ be the cell spectrum obtained by attaching the first $n$ cells of $X$. Then by induction, the motivic Adams spectral sequence converges for $X_{(n)} \wedge H \mathbb{Z} / p$. Consider the diagram

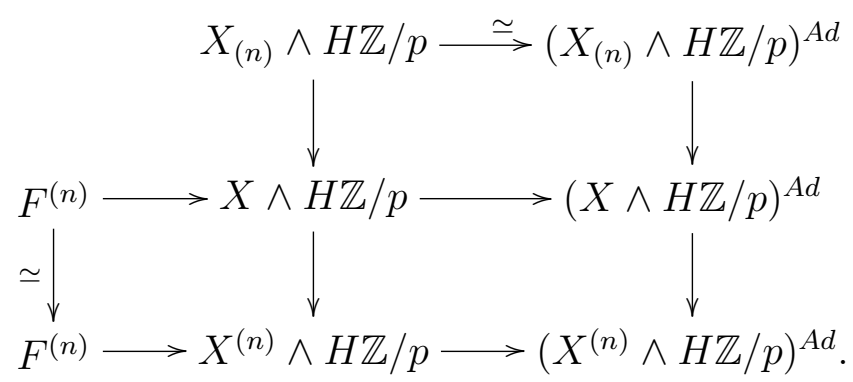

All the lines are cofiber sequences. By considering the bottom row and Lemma 11, the connectivity of $F^{(n)}$ goes to $\infty$ with $n$, while it does not depend on $n$ by the middle row. Thus, $F=F^{(n)} \simeq *$, as claimed.

Lemma 13. The spectrum $(S /(p, \eta))^{\text {Ad }}$ is very weakly equivalent to a cell spectrum of finite type. 
Proof: The proof mimics the analogous statement in topology. Let us first consider $p=2$. In topology, this spectrum is known as the first Brown-Gitler spectrum $B(1)$, and its Adams resolution is studied in [4], where it is shown that its connectivity (in total degree) is increasing. In the motivic setting, a similar argument can be made as follows.

Set

$$
B_{*}:=H \mathbb{Z} / 2_{*}(S /(2, \eta)) .
$$

Then $\left(H \mathbb{Z} / 2_{*}, B_{*}\right)$ is a sub-coalgebroid (in particular a bi-comodule) of $\left(H \mathbb{Z} / 2_{*}, A_{*}\right)$ via the map

$$
B_{*} \rightarrow A_{*}
$$

induced by the canonical map $S /(2, \eta) \rightarrow H \mathbb{Z} / 2$. Define now $J_{0}=A_{*}$, and define inductively short exact sequences of right $A_{*}$-comodules

$$
0 \rightarrow J_{m} \square_{A_{*}} B_{*} \rightarrow J_{m} \rightarrow J_{m+1} \rightarrow 0
$$

where the first map is the inclusion.

Claim: $\lim J_{m}=0$, and

$$
J_{m} \square_{A_{*}} B_{*}
$$

is isomorphic to a sum of copies of $B_{*}$.

The proof is the same as in topology: For example, filter $\left(H \mathbb{Z} / 2_{*}, A_{*}\right)$ by powers of the augmentation ideal. Then the associated graded object $\left(H \mathbb{Z} / 2_{*}, E_{0} A_{*}\right)$ is a (commutative) Hopf algebra, and isomorphic, as a coalgebra, to the tensor product of $\left(H \mathbb{Z} / 2_{*}, E_{0} B_{*}\right)$ with another coalgebra (where $E_{0} B_{*}$ is the associated graded object of $B_{*}$ with respect to the filtration by powers of its augmentation ideal). Therefore, the statement is true for the associated graded objects and hence also for the original objects (one shows by induction on $m$ that $\left.J_{m}\left(E_{0} A_{*}\right)=E_{0} J_{m}\right)$.

Noticing further that $J_{1}$ is concentrated in degrees $(\geq 2)+* \alpha$, the Claim implies by induction that there exists a resolution

$$
B_{*} \longrightarrow F_{0} \stackrel{d_{0}}{\longrightarrow} F_{1} \stackrel{d_{1}}{\longrightarrow} F_{2} \stackrel{d_{2}}{\longrightarrow} \cdots
$$

where $F_{i}$ is a sum of copies of $A_{*}$ on a finite set $S_{i}$ of generators $s$ in dimensions

$$
k_{s}=j+* \alpha, j \geq 2 i \text {. }
$$


As usual, to a Hopf algebroid extended/free resolution of the type (25), we may assign a tower of fibrations

$$
X_{i+1} \rightarrow X_{i} \rightarrow K_{i}
$$

where

$$
K_{i} \simeq \Sigma^{-i} \bigvee_{s \in S_{i}} \Sigma^{k_{s}} H \mathbb{Z} / 2
$$

and

$$
H \mathbb{Z} / 2_{*} X_{i}=\operatorname{Ker}\left(d_{i}\right), X_{0}=S /(2, \eta) .
$$

Further, by standard arguments, up to equivalence,

$$
\underset{n}{\operatorname{holim}} X_{n}
$$

does not depend on the choice of the resolution (25). In particular, for a suitable choice of resolution, (29) becomes $(S /(2, \eta))^{A d}$, and hence we expressed $(S /(2, \eta))^{A d}$ as a homotopy limit of a tower obtained by successively taking fibers of maps into $K_{i}$, so by $(26)$, (28), Lemma 8 and Lemma 12 applied to

$$
X=\Sigma^{-i} \bigvee_{s \in S^{i}} S^{k_{s}}
$$

$(S /(2, \eta))^{A d}$ is very weakly equivalent to a cell spectrum of finite type.

Now consider $p>2$. In this case, the above argument actually applies with $B(1)$ replaced by $M \mathbb{Z} / p$ (since $\xi_{1}$ is in dimension $(\geq 2)+$ $\ell \alpha)$. Thus, $M \mathbb{Z} / p^{A d}$ is very weakly equivalent to a cell spectrum of finite type, hence the same is true with $M \mathbb{Z} / p$ replaced by $S /(p, \eta)$, as $(?)^{A d}$ preserves cofibration sequences.

Remark: Note that we have not proved (yet) that the motivic Adams spectral sequence converges for $S /(p, \eta)$. However, we have the following

Lemma 14. For any 0-connective cell spectrum $X$ of finite type,

1. The canonical map $X /(p, \eta) \rightarrow(X /(p, \eta))^{\text {Ad }}$ is a 1-equivalence.

2. $(X /(p, \eta))^{A d}$ is very weakly equivalent to a cell spectrum of finite type.

3. The canonical map

$$
(X /(p, \eta))^{A d} \rightarrow\left((X /(p, \eta))^{A d}\right)^{A d}
$$

is a very weak equivalence. 
Proof: 1. For $X=S$, the characterization of the homotopy type of $(S /(p, \eta))^{A d}$ as a homotopy inverse limit of maps into the $K_{i}$ 's in the proof of Lemma 13, implies that the canonical map

$$
H \mathbb{Z} / 2 \rightarrow(S /(2, \eta))^{A d}
$$

for $p=2$, and

$$
H \mathbb{Z} / p \rightarrow(M \mathbb{Z} / p)^{A d}
$$

for $p>2$ is a 1-equivalence. Therefore, the statement follows from Lemma 10. Thus, by Lemma 9, the statement follows for the cell spectrum $X_{(n)}$ obtained by attaching the first $n$ cells of $X$. Now consider the diagram

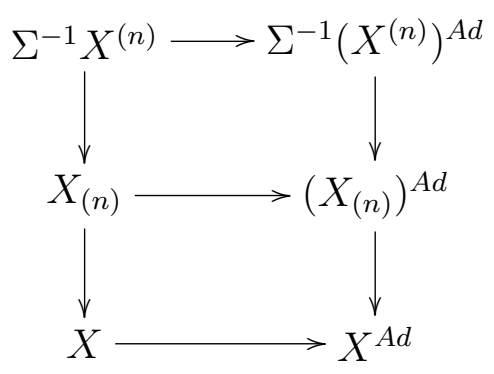

where the columns are cofibration sequences. By Lemma 11, for $n>>$ 0 , the first row is a map of 2-connective spectra, and hence is a 1equivalence. We just proved that the middle row is a 1-equivalence. Thus, the bottom row is a 1-equivalence by Lemma 9 .

2. Consider the right hand column of (30). The middle term is very weakly equivalent to a cell spectrum of finite type by Lemma 13, while the connectivity of the top term goes to $\infty$ with $n$ by Lemma 11. Thus, our statement follows from Lemma 8.

3. Using Lemma 11 again, it suffices to prove the statement for $X=S$. we go back to the model

$$
(S /(p, \eta))^{A d}=\underset{n}{\operatorname{holim}} Y_{n}
$$

where $Y_{n}$ are the cofibers

$$
X_{n} \rightarrow S /(p, \eta) \rightarrow Y_{n}
$$

(see $(27)$ ). The $Y_{n}$ 's are obtained by taking successive fibers of maps into the spectra $K_{i}$ of increasing connectivity. Since the motivic Adams spectral sequence converges for $K_{i}$ by (a special case of) Lemma 12, it converges for $Y_{n}$. Since the connectivity of the $X_{n}$ 's goes to $\infty$ with $n$, our statement follows from Lemma 11. 
Remark: Note that since $X /\left(p^{M}, \eta^{N}\right)$ may be represented by a motivic spectrum with a finite filtration where the associated graded pieces are $X /(p, \eta)$, all the statements of Lemma 14 remain valid with $X /(p, \eta)$ replaced by $X /\left(p^{M}, \eta^{N}\right)$.

Lemma 15. Let $X$ be a cell spectrum of finite type and suppose there exists an $N$ such that

$$
p^{N}=\eta^{N}=0: X \rightarrow X .
$$

Then the motivic Adams spectral sequence converges for $X$.

Proof: Suppose without loss of generality that $X$ is 0 -connective. Using (31), there exists an $M_{1}, N_{1}$ such that

$$
X /\left(p^{M_{1}}, \eta^{N_{1}}\right) \simeq X \vee \Sigma X \vee \Sigma^{1+\alpha} X \vee \Sigma^{2+\alpha} X
$$

(Choosing $M_{1} \geq N$ makes $X / p^{M} \simeq X \vee \Sigma X$. By choosing another, possibly larger, number $N_{1}, 0=\eta^{N_{1}}: X /\left(p^{M_{1}}\right) \rightarrow X /\left(p^{M_{1}}\right)$.) Then by Lemma 14 1., the canonical map

$$
X /\left(p^{M_{1}}, \eta^{N_{1}}\right) \rightarrow\left(X /\left(p^{M_{1}}, \eta^{N_{1}}\right)\right)^{A d}=X^{A d} /\left(p^{M_{1}}, \eta^{N_{1}}\right)
$$

is a 1-equivalence, while by Lemma 14 2., the right hand side is very weakly equivalent to a cell spectrum of finite type, for which, further, the motivic Adams spectral sequence converges by Lemma 14, 3. Thus, the fiber $X_{1}$ of (33) is 1-connective, and satisfies again the conditions of Lemma 15.

Therefore, by iterating this procedure, for any chosen $k$, we may construct a wedge $X^{\prime}$ of $X$ and some suspensions of the form $\Sigma^{(\geq 1)+\ell \alpha} X$ such that

$$
X^{\prime} \rightarrow\left(X^{\prime}\right)^{A d}
$$

is a $k$-equivalence. But a wedge summand of a $k$-equivalence is a $k$ equivalence, so

$$
X \rightarrow X^{A d}
$$

is also a $k$-equivalence for any $k$, and hence a very weak equivalence.

Our next step is proving an analogue of Lemma 23 of [9]. Because we are no longer working over an algebraically closed field, it is more involved, and we need some preliminary steps. 
Lemma 16. Suppose $X$ is a motivic spectrum over $F$, and

$$
X=\operatorname{holim} X_{s}
$$

where each motivic spectrum $X_{s}$ is of bounded type with the same bounds $\left(N_{m}\right)_{m \in \mathbb{Z}}$. Assume one of the following conditions hold:

(1) There exists a prime $p>2$ and a number $N \in \mathbb{N}$ such that

$$
0=p^{N}: X_{s} \rightarrow X_{s}
$$

for each $s$. Further, -1 is a sum of squares in $F$, and $c d_{p}(F)<$ $\infty$.

(2) There exists an $N \in \mathbb{N}$ such that

$$
0=2^{N}=[-1]^{N}: X_{s} \rightarrow X_{s}
$$

For each s. Further, $c d_{2}(F[i])<\infty$.

Then there exists a very weak equivalence

$$
Y \rightarrow X
$$

where $Y$ is a cell motivic spectrum of bounded type.

Proof: By induction on $i$, we will construct an $i$-equivalence

$$
Y_{i} \rightarrow X
$$

where $Y_{i}$ is a cell spectrum of bounded type and there exists a number $N_{i} \in \mathbb{N}$ such that

$$
0=p^{N_{i}}: Y_{i} \rightarrow Y_{i}
$$

in Case (1) and

$$
0=2^{N_{i}}=[-1]^{N_{i}}: Y_{i} \rightarrow Y_{i}
$$

in Case (2).

If $k$ is such that $N_{m}=\infty$ for $m \leq k$, we may put $Y_{k}:=*$. Now suppose (35) has been constructed for a given $i$. Let $X_{i+1}$ be the homotopy cofiber of (35). Then

$$
\pi_{m+\ell \alpha} X_{i+1}=0 \text { for } m \leq i .
$$

Furthermore,

$$
X_{i+1}=\operatorname{holim} X_{i+1, s}
$$

where the motivic spectra $X_{i+1, s}$ are $(i+1)$-connective, bounded below with the same bounds, and annihilated by the same power of $p$ in Case $(1)$, and the same power of $2,[-1]$ in Case (2). 
Now note however that the condition (1) implies that for each $k \in \mathbb{N}$, there exists an $N \in \mathbb{Z}$ such that

$$
\pi_{\ell \alpha} M \mathbb{Z} /\left(p^{k}\right)=0 \text { for } \ell<N
$$

and condition (2) implies that for each $k \in \mathbb{N}$ there exists an $N \in \mathbb{Z}$ such that

$$
\pi_{\ell \alpha} S /\left(2^{k},[-1]^{k}\right)=0 \text { for } \ell<N .
$$

By Lemma 7 , then, there is an $N \in \mathbb{Z}$ such that

$$
\pi_{i+1+\ell \alpha} X_{i+1, s}=0 \text { for } \ell<N .
$$

Hence,

$$
\pi_{i+1+\ell \alpha} X_{i+1}=0 \text { for } \ell<N .
$$

Thus, by attaching cells of dimension $i+1+\ell \alpha, \ell \geq N$, to $X_{i+1}$, we may kill $\pi_{i+1+* \alpha} X_{i+1}$. Further, the attaching map will factor through the smash product with $M \mathbb{Z} / p^{K}$ for some $K$ in Case (1), and with $S /\left(2^{K_{1}},[-1]^{K_{2}}\right)$ with some $K_{2}>K_{1}>0$ (since smashing $X_{i+1}$ with such spectra will produce a wedge sum of copies of $X_{i+1}$ ).

Composing these attaching maps with the connecting map $\Sigma^{-1} X_{i+1} \rightarrow$ $Y_{i}$ of (35) and taking homotopy cofiber produces $Y_{i+1}$.

Recall now that there is a $t$-structure on the (triangulated) homotopy category of cell motivic spectra where $\tau_{\leq 0}$ is obtained by attaching cells in a way so as to kill homotopy groups in dimensions $k+\ell \alpha, k>0$. The heart $\mathcal{C}$ is equivalent to the category of $K_{M W}$-modules.

Lemma 17. Let $M \in O b j \mathcal{C}$ and suppose that either

$$
\begin{aligned}
& p>2,-1 \text { is a sum of squares in } F, \\
& c d_{p}(F)<\infty \text { and } 0=p^{N}: M \rightarrow M
\end{aligned}
$$

or

$$
\begin{aligned}
& c d_{2}(F)<\infty \text { and } 0=2^{N}=[-1]^{N}: M \rightarrow \\
& M .
\end{aligned}
$$

Then $M$ is equivalent to a cell spectrum of bounded type.

Proof: The point is we may form a "resolution" of the form

$$
F_{i} \rightarrow M_{i} \rightarrow M_{i+1}
$$

where $M_{0}=M, F_{i}, M_{i} \in \operatorname{Obj}\left(\Sigma^{i} \mathcal{C}\right)$, the first map in (42) is surjective on homotopy groups and such that $\Sigma^{-i} F_{i}$ is a direct sum of copies of

$$
K_{M W} / p^{N}
$$


in Case (40) and of

$$
K_{M W} /\left(2^{N},[-1]^{N}\right)
$$

in Case (41). Thus, it suffices to prove the statement for (43) in Case (40) and (44) in Case (41).

The idea now is to apply Lemma 16 to $X$ of the form (43) resp. (44), $X_{s}=X / \eta^{s}$. The spectra $X_{s}$ can be constructed can be constructed by taking finitely many cofibers of (suspensions of) copies of $K_{M W} /(\eta, p)=K_{M} / p$.

Thus, it suffices to show that $K_{M} / p$ is of bounded type. For $p=2$, we have a cofibration

$$
\Sigma^{1-\alpha} H \mathbb{Z} / 2 \rightarrow H \mathbb{Z} / 2 \rightarrow K_{M} / 2
$$

(the first map being the Tate twist), so this follows from $H \mathbb{Z} / 2$ being of finite type. For $p>2, c d_{p}(F)<\infty$ and the Bloch-Kato conjecture [18] imply that there exists an $N \in \mathbb{Z}$ such that $H \mathbb{Z} / p_{m+n \alpha}=0$ for $m+n<N$. It follows that constructing $K_{M} / p$ from $H \mathbb{Z} / p$ by killing off homotopy groups $\pi_{k+* \alpha}, k \geq 1$ by attaching cells in the category of (rigid) $H \mathbb{Z} / p$-modules produces a finite type $H \mathbb{Z} / p$-module and hence a finite type (motivic) spectrum.

Lemma 18. Let $X$ be a motivic spectrum of bounded type over $F$, and suppose one of the following conditions holds:

$$
\begin{aligned}
& p>2,-1 \text { is a sum of squares in } F, \\
& c d_{p}(F)<\infty \text { and } 0=p^{N}: X \rightarrow X
\end{aligned}
$$

or

$$
\begin{aligned}
& c d_{2}(F)<\infty \text { and } 0=2^{N}=[-1]^{N}: X \rightarrow \\
& X .
\end{aligned}
$$

Then $\pi_{*} X$ is of bounded type.

Proof: The strategy is to consider the canonical (co)fibration sequences

$$
\tau_{\geq i+1} X \rightarrow \tau_{\geq i} X \rightarrow Y_{i}
$$

and notice that by induction on $i, \tau_{\geq i} X$ satisfy the assumptions of this Lemma, while $Y_{i}$ satisfy the assumptions of Lemma 17. (Start with $i=k$ where $X$ is $k$-connective.)

Next, notice that by our assumptions, the fact that $\tau_{\geq i} X$ is of bounded type and by Lemma 7 , for each $i$ there exists an $N_{i}$ such that $\pi_{i+\ell \alpha} \tau_{\geq i} X=$ 0 for $\ell<N_{i}$. 
Lemma 19. When $p>2,-1$ is a sum of squares in $F$ and $c d_{p}(F)<$ $\infty$, or $p=2$ and (4) holds, the canonical map

$$
\Psi: M \mathbb{Z} / p \rightarrow \underset{n}{\operatorname{holim}}(M \mathbb{Z} / p) / \eta^{n}
$$

is a very weak equivalence.

Proof: Similarly as in [9], the fiber $F \Psi$ of $\Psi$ is the homotopy inverse limit of

$$
\cdots \longrightarrow \Sigma^{(k+1) \alpha} M \mathbb{Z} / p \stackrel{\eta}{\longrightarrow} \Sigma^{k \alpha} M \mathbb{Z} / p \longrightarrow \cdots .
$$

Let us first assume that $p>2$. Then, by [18], there exists a constant $N$ such that

$$
\left(K_{M}(F) / p\right)_{n}=0 \text { for } n>N .
$$

Then we will prove that for all $q \in \mathbb{Z}$ there exists an $\ell \geq 0$ such that

$$
\pi_{q} \Sigma^{k \alpha} M \mathbb{Z} / p=0 \text { for } k \geq \ell,
$$

hence proving our statement, since this implies

$$
\lim _{\leftarrow} \pi_{q} \Sigma^{k \alpha} M \mathbb{Z} / p=\lim _{\leftarrow}{ }^{1} \pi_{q} \Sigma^{k \alpha} M \mathbb{Z} / p=0 .
$$

In effect, by Lemma $18, \pi_{*} M \mathbb{Z} / p$ is of bounded type, which implies (50).

To treat the case when $p=2$ and (4) is satisfied, first observe that by Lemma 18 applied to $X=S /(p,[-1])$

$$
\pi_{*+* \alpha}(F \Psi /[-1])=0 .
$$

Therefore,

$$
[-1]: \pi_{*+* \alpha}(F \Psi) \rightarrow \pi_{*+* \alpha}(F \Psi)
$$

is an isomorphism. Since an isomorphism of these groups is also induced by $\eta$, the same is true for $[-1] \eta^{2}$, but $[-1] \eta^{2}=0 \in K_{M W}(F) / 2$. Thus, $\pi_{*+* \alpha} F \Psi=0$, as claimed.

Comment: It may seem that in the case $p=2$, condition (4) could be replaced by a weaker condition, replacing [-1] by any element (or sequence of elements) which, when multiplied by $\eta$, become nilpotent in $K_{M W} / 2$. Note however that by Morel's structure theorem $(6)[14,13]$,

$$
\begin{array}{r}
K_{M W} / 2=W / 2 \text { in dimensions }(\geq 0) \alpha \\
I^{n} / 2 \text { in dimensions }(\leq 0) \alpha
\end{array}
$$

where $I$ is the augmentation ideal of the Witt ring. ( $\operatorname{In}(6), I^{-n} / I^{-n+1} \cong$ $\left(K_{M}\right)_{n} / 2$, so modulo 2 , the right hand leg of the pullback becomes an isomorphism.) Thus, an element $\alpha$ annihilates $\eta$ in $K_{M W} / 2$ if and 
only if $\alpha$ represents an element of $W$ which is divisible by 2 . But $2=[-1] \in W$, so this means that $\alpha$ is divisible by $[-1]$, showing that essentially no further generalization is meaningful.

Lemma 20. Let $X$ be a motivic cell spectrum of finite type, and let either $p>2, c_{p}(F)<\infty$ and -1 be a sum of squares in $F$ or $p=2$ and $F$ satisfy the condition (4). Then for any $k$, the canonical map

$$
X / p^{k} \rightarrow \underset{n}{\operatorname{holim}} X /\left(p^{k}, \eta^{n}\right)
$$

is a very weak equivalence.

Proof: Let, again, $X_{(m)}$ be the spectrum obtained by attaching the first $m$ cells of $X$. Consider the diagram of cofibration sequences

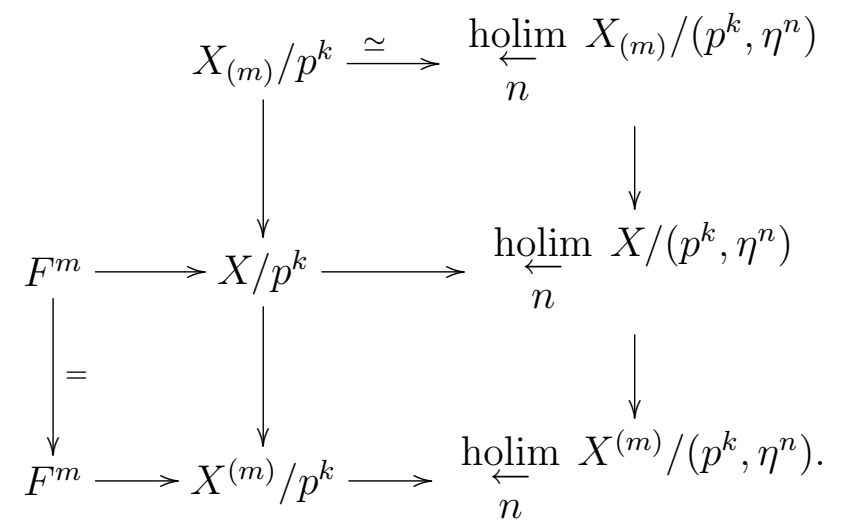

The top row is an equivalence by Lemma 19. The connectivity of $F^{m}$ goes to $\infty$ with $m$ by considering the botom row, but does not depend on $m$ by considering the middle row. Thus, $F=F^{m} \simeq *$, as claimed.

Proof of Theorem 1: The first statement follows directly from Lemma 15. The second statement follows then from Lemma 20.

\section{REFERENCES}

[1] J.F. Adams: Stable homotopy and generalised homology, Chicago Lectures in Mathematics, University of Chicago Press, 1973

[2] J. Arason: Cohomologische invarianten quadratischer Formen, J. Algebra 36 (1975) 448-491

[3] A.K.Bousfield, D.M.Kan: Homotopy limits, completions and localizations, Lecture Notes in Mathematics 304, Springer Verlag, 1972 
[4] E.H.Brown, R.L.Cohen: The Adams spectral sequence of $\Omega^{2} S^{3}$ and BrownGitler spectra, in: Algebraic Topology and Algebraic K-theory, W.Browder, ed., Annals of Math. Studies 113, Princeton Univ. Press, Princeton, NJ, 1987, pp. $101-125$

[5] A.K.Bousfield: The localization of spectra with respect to homology, Topology 18 (1979) 257-281

[6] D.Dugger, D.Isaksen: The motivic Adams spectral sequence, arXiv:0901.1632

[7] D.Dugger, D.Isaksen: Motivic cell structures, Algebr. Geom. Topol. 5 (2005) 615-652

[8] M.Hill: Ext and the motivic Steenrod algebra over $\mathbb{R}$, to appear in J. Pure and Appl. Alg.

[9] P.Hu, I.Kriz, K.Ormsby: Remarks on motivic homotopy theory over algebraically closed fields, J. K-theory, doi: 10.1017/is010001012jkt098

[10] P.Hu, I.Kriz, K.Ormsby: Equivariant and real motivic stable homotopy theory, $K$-theory archive 952

[11] T. Y. Lam. Introduction to quadratic forms over fields, volume 67 of Graduate Studies in Mathematics. American Mathematical Society, Providence, RI, 2005.

[12] J.Milnor: Algebraic K-theory and quadratic forms, Invent. Math. 9 (1969/1970) 318-344

[13] F.Morel: On the motivic stable $\pi_{0}$ of the sphere spectrum, Axiomatic, Enriched and Motivic Homotopy Theory, pp. 219-260, 2004 Kluwer Academic Publishers

[14] F.Morel: An introduction to $\mathbb{A}^{1}$-homotopy theory, Contemporary developments in algebraic K-theory, ICTP Lect. Notes, XV (2004) 357-441

[15] P.A.Østvær, O. Röndigs: Rigidity in motivic homotopy theory, Math. Ann. 341 (2008) 651-675

[16] K.Ormsby: Motivic invariants of $p$-adic fields, to appear

[17] J.P.Serre: Galois Cohomology, Springer Monographs in Mathematics, Springer-Verlag, Berlin, 2002

[18] V.Voevodsky: On Motivic Cohomology with $\mathbb{Z} / \ell$ coefficients, arXiv:0805.4430

[19] V.Voevodsky: Motivic cohomology with Z/2-coefficients, Publ. Math. Inst. Hautes Études Sci. 98 (2003) 59-104

[20] V.Voevodsky: Reduced power operations in motivic cohomology, Publ. Math. Inst. Hautes Études Sci. 98 (2003) 1-57

[21] V.Voevodsky: Motivic Eilberg-Maclane spaces, arXiv:0805.4432 